\title{
Quantitative physiology and elemental composition of Kluyveromyces lactis CBS 2359 during growth on glucose at different specific growth rates
}

\author{
Oscar Dias • Thiago O. Basso - Isabel Rocha • Eugénio C. Ferreira • \\ Andreas K. Gombert
}

Received: 3 May 2017/Accepted: 5 September 2017/Published online: 12 September 2017

(C) Springer International Publishing AG 2017

\begin{abstract}
The yeast Kluyveromyces lactis has received attention both from academia and industry due to some important features, such as its capacity to grow in lactose-based media, its safe status, its suitability for large-scale cultivation and for heterologous protein synthesis. It has also been considered as a model organism for genomics and metabolic regulation. Despite this, very few studies were carried out hitherto under strictly controlled conditions, such as those found in a chemostat. Here we report a set of quantitative physiological data generated during chemostat cultivations with the $K$. lactis CBS 2359 strain, obtained under glucose-limiting and fully aerobic conditions. This dataset serve as a basis for the comparison of $K$. lactis with the model yeast Saccharomyces cerevisiae in terms of their elemental
\end{abstract}

Oscar Dias and Thiago O. Basso contributed equally to this work.

O. Dias · I. Rocha - E. C. Ferreira

Centre of Biological Engineering, University of Minho, Campus de Gualtar, 4710-057 Braga, Portugal

O. Dias · T. O. Basso $(\bowtie) \cdot$ A. K. Gombert Department of Chemical Engineering, Polytechnic School, University of São Paulo, Av. Prof. Luciano Gualberto 380, São Paulo, SP 05508-010, Brazil e-mail: thiagobasso@usp.br

\section{A. K. Gombert}

School of Food Engineering, University of Campinas, Rua Monteiro Lobato 80, Campinas, SP 13083-862, Brazil compositions, as well as for future metabolic flux analysis and metabolic modelling studies with $K$. lactis.

Keywords Kluyveromyces lactis · Yeast physiology $\cdot$ Chemostat cultivation $\cdot$ Elemental composition

\section{Introduction}

The yeast Kluyveromyces lactis has long been considered a suitable microbe for industrial applications, mainly aiming at the production of heterologous proteins (Blondeau et al. 1993; Hensing et al. 1995; Walsh et al. 1998; van Ooyen et al. 2006; Rocha et al. 2011; Spohner et al. 2016), which is mainly due to: i) its safe status (Bonekamp and Oosterom 1994); ii) its high capacity of converting sugars into biomass (Kiers et al. 1998); iii) its suitability for large-scale cultivation (Hensing et al. 1995); and iv) its capacity of growing on lactose-based media (Dickson et al. 1979; Dickson and Markin 1980; Dickson and Barr 1983).

$K$. lactis may also be considered a model organism (Schaffrath and Breunig 2000), which has been investigated by the scientific community to elucidate molecular mechanisms such as transcriptional regulation (Hoekstra et al. 1994; Becerra et al. 2004; Suleau et al. 2006), the Crabtree effect (Breunig et al. 2000; González-Siso et al. 2000), oxidative stress response 
(González-Siso et al. 2009) and even in cell aging studies (Oliveira et al. 2008; Roux et al. 2010), among others. Furthermore, it has been included in evolutionary genomics studies of yeasts (Dujon et al. 2004; Souciet et al. 2009). Already some decades ago, the strain CBS 2359 was chosen as a model strain (Wésolowski-Louvel et al. 1996), in order to facilitate comparison among results obtained in different laboratories, so as to increase the gaining of knowledge using this organism as a model. A dedicated conference series took place until 2005 (Clark-Walker 2006) and some years later, a whole issue (August, 2007) of the FEMS Yeast Research journal was dedicated to $K$. lactis (Fukuhara and Boekhout 2007), covering varied molecular and physiological aspects of this species.

At the beginning of the 21 st century, the whole Kluyveromyces taxon was redefined (Kurtzman 2003). Some species that had been part of the original genus Kluyveromyces since its creation were transferred to new genera (e.g. Kluyveromyces polysporus), whereas others remained within the genus Kluyveromyces. Interestingly, although the species Kluyveromyces marxianus was proposed as the type species of the newly defined genus, $K$. lactis continues to receive more attention from scientists than its sister species (Fonseca et al. 2008; Lane and Morrissey 2010). It was not until Kiers et al. (1998) performed detailed quantitative chemostat cultivations in controlled bioreactors that the Crabtree-negative aspect of this yeast was clarified. While it was demonstrated that oxygen limitation is the primary trigger for alcoholic fermentation (as long as the carbon and energy source is the sole growth-limiting substrate), it could also be established that the so-called short-term Crabtree effect (i.e. ethanol formation after exposure of respiring cells to a sudden glucose excess under fully aerobic conditions) also occurs in this species.

In spite of the frequent use of $K$. lactis in molecular studies, as mentioned above, there is a limited number of reports on the cultivation of this organism in controlled bioreactors using continuous culture. This type of experiment is required, when one aims at investigating microbial physiology in a quantitative manner. Here, we report a set of quantitative physiological data generated during chemostat cultivations with the $K$. lactis CBS 2359 strain, obtained under fully aerobic conditions. Our data basically confirm those reported previously by Kiers et al. (1998), which is per se an important feature [Nature, editorial,
August 24, (2016)], but we also measured the elemental composition of the cellular biomass at different growth rates (=dilution rates). Besides this novelty, these data serve as a basis for the comparison of $K$. lactis with the model yeast Saccharomyces cerevisiae in terms of their elemental composition, as well as for future metabolic flux analysis and metabolic modelling studies with $K$. lactis (Dias et al. 2014).

\section{Methods}

Yeast strain and maintenance

The yeast strain $K$. lactis CBS 2359 (ATCC 8585/DSM 70799/NBRC 1267/NRRL Y-1140/ WM37) was obtained from the Centraalbureau voor Schimmelcultures, Utrecht, The Netherlands, and used throughout this study. Stock cultures were prepared by growing cells in shake flasks containing $100 \mathrm{~mL}$ YP medium (1\% yeast extract, $2 \%$ bacteriological peptone) with $20 \mathrm{~g} \mathrm{~L}^{-1}$ initial glucose. After overnight growth at $30{ }^{\circ} \mathrm{C}$ and $200 \mathrm{rpm}, 20 \%$ (final concentration, v/v) glycerol was added and $1 \mathrm{~mL}$ aliquots were stored at $-80{ }^{\circ} \mathrm{C}$. Stock cultures were used to prepare pre-cultures for batch and chemostat cultivations in bioreactors.

\section{Cultivation medium}

The composition of the defined medium used during batch and chemostat cultivations in bioreactors was the one described by Verduyn et al. (1992), with a single modification: nicotinic acid concentration was increased five times, as proposed by Kiers et al. (1998). The medium contained (in $\left.\mathrm{g} \mathrm{L}^{-1}\right)$ : $\left(\mathrm{NH}_{4}\right)_{2-}$ $\mathrm{SO}_{4}, 5.0 ; \mathrm{KH}_{2} \mathrm{PO}_{4}, 3.0 ; \mathrm{MgSO}_{4} \cdot 7 \mathrm{H}_{2} \mathrm{O}, 0.5$; and trace elements consisting of $\left(\mathrm{mg} \mathrm{L}^{-1}\right)$ EDTA, $15, \mathrm{ZnSO}_{4}$ $7 \mathrm{H}_{2} \mathrm{O}, 4.5, \mathrm{MnCl}_{2} \cdot 2 \mathrm{H}_{2} \mathrm{O}, 0.84 ; \mathrm{CoCl}_{2} \cdot 6 \mathrm{H}_{2} \mathrm{O}, 0.3$; $\mathrm{CuSO}_{4} \cdot 5 \mathrm{H}_{2} \mathrm{O}, 0.3 ; \mathrm{Na}_{2} \mathrm{MoO}_{4} \cdot 2 \mathrm{H}_{2} \mathrm{O}, 0.4 ; \mathrm{CaCl}_{2} \cdot 2 \mathrm{H}_{2} \mathrm{O}$, 4.5, $\mathrm{FeSO}_{4} \cdot 7 \mathrm{H}_{2} \mathrm{O}, 3.0, \mathrm{H}_{3} \mathrm{BO}_{3}, 1.0, \mathrm{KI}, 0.1$. A solution containing vitamins was filter-sterilized and added to the medium to a final concentration of $\left(\mathrm{mg} \mathrm{L}^{-1}\right)$ d-biotin, 0.05; calcium pantothenate, 1.0; nicotinic acid, 5.0; myo-inositol, 25; thiamine $\mathrm{HCl}, 1.0$; pyridoxine $\mathrm{HCl}, 1.0$, and para-aminobenzoic acid, 0.20. 
Batch cultivations in a bioreactor

Precultures for batch bioreactor cultivations were grown overnight in an orbital shaker at $30{ }^{\circ} \mathrm{C}$ and $200 \mathrm{rpm}$ in $500 \mathrm{~mL}$ shake flasks containing $100 \mathrm{~mL}$ of the defined medium with $20 \mathrm{~g} \mathrm{~L}^{-1}$ initial glucose. Batch cultivations were conducted in a BioFlo III (New Brunswick Scientific) bioreactor, with $4 \mathrm{~L}$ working volume. The same defined medium was used, with $20 \mathrm{~g} \mathrm{~L}^{-1}$ initial glucose as the sole carbon and energy source. The medium was supplemented with $0.2 \mathrm{~g} \mathrm{~L}^{-1}$ antifoam Emulsion C (Sigma, St. Louis, MO), pH was controlled at 5.0 by automatic addition of $2 \mathrm{M} \mathrm{KOH}$, and the temperature was kept constant at $30{ }^{\circ} \mathrm{C}$. Dissolved oxygen concentration was kept above $60 \%$ initial saturation with air by manually adjusting the agitation rate between 700 and $850 \mathrm{rpm}$, and by keeping the airflow into the reactor at $4 \mathrm{~L} \mathrm{~min}^{-1}$. Samples were taken periodically and analysed for the concentration of biomass (indirectly via absorbance measurements) and of metabolites (via HPLC), as well as for $\mathrm{pH}$.

\section{Chemostat cultivations in a bioreactor}

Precultures for chemostat cultivations were grown overnight in an orbital shaker at $30{ }^{\circ} \mathrm{C}$ and $200 \mathrm{rpm}$ in $500 \mathrm{~mL}$ shake flasks containing $100 \mathrm{~mL}$ of the defined medium with $10 \mathrm{~g} \mathrm{~L}^{-1}$ initial glucose. Cultivations were conducted in a BioFlo III bioreactor (New Brunswick Scientific), as described elsewhere (Basso et al. 2014). The feeding vessel contained the same defined medium with $10 \mathrm{~g} \mathrm{~L}^{-1}$ glucose as the sole carbon and energy source. The medium was also supplemented with $0.2 \mathrm{~g} \mathrm{~L}^{-1}$ antifoam Emulsion C (Sigma, St. Louis, MO). Cultivations were carried out under the following conditions: working volume of $1 \mathrm{~L}, \mathrm{pH}$ controlled at 5.0 by automatic addition of $2 \mathrm{M}$ $\mathrm{KOH}$, and constant temperature of $30^{\circ} \mathrm{C}$. Agitation was manually kept between 700 and $925 \mathrm{rpm}$, and the air flow into the reactor was in the range of 0.5 to $1 \mathrm{~L} \mathrm{~min}^{-1}$; these conditions kept the dissolved $\mathrm{O}_{2}$ concentration always above $60 \%$ of air saturation (full aerobiosis), as indicated by a dissolved oxygen electrode. Chemostat cultivations were preceded by a batch growth phase, carried out under the same conditions as those described above. The switch from batch to continuous cultivation mode was made when a sharp drop in the off gas $\mathrm{CO}_{2}$ signal was observed, indicating carbon source exhaustion. The cultures were assumed to be in steady state when, after at least five volume changes without altering the cultivation conditions, the culture dry mass and the specific $\mathrm{CO}_{2}$ production rate varied less than $2 \%$ over two additional volume changes. Steady state situations were achieved for four different dilution rates $(D)$ in each experiment: $0.1,0.2,0.3$, and $0.4 \mathrm{~h}^{-1}$. Continuous cultures were performed in duplicate. The exhaust gas from the bioreactor was cooled in a condenser $\left(2{ }^{\circ} \mathrm{C}\right)$ to minimize ethanol evaporation and subsequently dried in silica-filled flasks, before being conveyed into the offgas analyser (Applikon Biotechnology, Schiedam), which detected the molar fractions of $\mathrm{O}_{2}$ and $\mathrm{CO}_{2}$. Samples were taken in the different steady states for the analysis of biomass concentration (in terms of the culture dry mass), metabolite concentration (via HPLC), $\mathrm{pH}$, and elemental composition of biomass.

Determination of biomass concentration and concentrations of extracellular metabolites

Culture supernatants were obtained by centrifugation and used for determining the concentrations of sugars (glucose), organic acids, ethanol, and glycerol. In the case of residual sugar concentration analysis, rapid sampling from the bioreactor with cold steel beads was employed, as described by Mashego et al. (2003). The compounds were separated by HPLC using an HPX$87 \mathrm{H}$ ion-exchange column (Bio-Rad) at $60{ }^{\circ} \mathrm{C}$, with $5 \mathrm{mM} \mathrm{H}_{2} \mathrm{SO}_{4}$ as the mobile phase, at a flow rate of $0.6 \mathrm{~L} \mathrm{~min}^{-1}$. Glucose, ethanol, acetate, glycerol, succinate, and lactate were detected using a Waters 2414 refractive index detector; pyruvate was detected using a Waters $2487 \mathrm{UV}$ detector at $214 \mathrm{~nm}$.

During batch cultivations, biomass concentration was indirectly assessed using absorbance measurements performed on culture samples, using a Genesys 20 (Thermo Scientific) spectrophotometer, at $600 \mathrm{~nm}$.

During chemostat cultivations, the dry mass of the biomass was determined gravimetrically, in duplicate, by filtering a $5 \mathrm{~mL}$ culture sample through a $0.45 \mu \mathrm{m}$ filter membrane. The filter was subsequently washed with distilled water, dried in a microwave oven and weighed (Olsson and Nielsen 1997).

Elemental composition of cell biomass

Culture samples were first collected in an ice bath from the bioreactor outlet, centrifuged and lyophilized. The 
carbon, nitrogen and hydrogen contents were determined using an Elemental Analyzer $2400 \mathrm{CHN}$ (Perkin Elmer). Briefly, the sample was first weighed using a six decimal digit balance and then introduced into the equipment, where combustion occurs in the presence of pure oxygen. In this way, all carbon in the sample is converted to $\mathrm{CO}_{2}$, hydrogen to $\mathrm{H}_{2} \mathrm{O}$ and nitrogen into various oxides $\left(\mathrm{N}_{\mathrm{X}} \mathrm{O}_{\mathrm{X}}\right)$. The latter are forced into a column where reduction into $\mathrm{N}_{2}$ occurs with $\mathrm{Cu}^{0}$. The $\mathrm{CO}_{2}, \mathrm{H}_{2} \mathrm{O}$ and $\mathrm{N}_{2}$ gases are dragged by $\mathrm{He}$ gas and separated in a chromatographic column and detected via a thermal conductivity detector (TCD).

The sulphur and phosphorous contents in dried biomass samples were analyzed in the Spectro Arcos SOP (Spectro) equipment. Briefly, the sample was digested in a fully closed Schoniger flask, in which all sulphur is converted into $\mathrm{SO}_{4}$ and all phosphorous into $\mathrm{PO}_{4}$. Subsequently, these gases were absorbed in water and diluted in volumetric flasks. The technique employed in the quantification was Inductively coupled plasma atomic emission spectroscopy (ICPAES).

For the ash content determination, the lyophilized biomass samples were first fully dried in an oven at $75^{\circ} \mathrm{C}$ for $4 \mathrm{~h}$ using pre-dried ashing crucibles, weighed and subsequently treated by the procedure described by Sluiter et al. (2005). Briefly, the fully dried samples (in the crucibles) were treated in a Quimis 318D Muffle furnace (Quimis, Brazil) using the following temperature profile: $105^{\circ} \mathrm{C}$ for $12 \mathrm{~min}$, $250{ }^{\circ} \mathrm{C}$ for $30 \mathrm{~min}$ and $575^{\circ} \mathrm{C}$ for $180 \mathrm{~min}$. After that, samples were cooled to room temperature in a desiccator and weighted for ash determination.

\section{Data consistency analysis}

Steady-state specific rates were analysed for data consistency using the software MACROBAL 2.02, obtained from the Delft University of Technology (Hellinga and Romein 1992). In order to calculate the estimated conversion rates, elemental composition of cell biomass for each dilution rate was provided to the software, as well as the experimental specific rates for biomass and carbon dioxide production, and for oxygen and glucose consumption.

\section{Results}

Batch bioreactor cultivations

A batch cultivation was carried out in duplicate, using a bioreactor with a $4 \mathrm{~L}$ working volume, as described in the "Methods" section. The initial glucose concentration was $20 \mathrm{~g} \mathrm{~L}^{-1}$. The cultivations were interrupted when the glucose concentration was around $12 \mathrm{~g} \mathrm{~L}^{-1}$ (around $11 \mathrm{~h}$ cultivation time), before any oxygen or another nutrient limitation eventually set in. Kiers et al. (1998) performed similar experiments, except for the fact that the initial glucose concentration was $10 \mathrm{~g} \mathrm{~L}^{-1}$ in their experiments, which already required blending of air with pure oxygen during the final hour of the experiment, to avoid the dissolved oxygen concentration to drop below $40 \%$ of initial saturation with air.

A clear exponential growth phase was observed in both cultivations. Since samples were taken every hour, a maximum specific growth rate of $0.45 \pm 0.01 \mathrm{~h}^{-1}$ (deviation of the mean) could be calculated, using nine datapoints in each cultivation (results not shown). This value is very close to the $0.48 \mathrm{~h}^{-1}$ value reported by Kiers et al. (1998) for the same strain and growth conditions, and also to the $0.465 \mathrm{~h}^{-1}$ value reported by Inchaurrondo et al. (1998) for a different strain (NRRL Y-1118) and a slightly different defined medium. In order to keep the dissolved oxygen concentration above $60 \%$ of initial saturation with air along the entire cultivation, the agitation speed needed to be manually increased from the initial $700 \mathrm{rpm}$ value, up to $850 \mathrm{rpm}$, during the final period (from 9 to $11 \mathrm{~h}$ ) of the batch cultivations. No ethanol formation was observed during these cultivations, however, glycerol accumulation occurred to levels up to $0.2 \mathrm{~g} \mathrm{~L}^{-1}$. Inchaurrondo et al. (1998) did not report on metabolite formation during batch cultures in their work, whereas Kiers et al. (1998) reported that ethanol concentrations remained below detection limit during the entire batch cultivation with K. lactis CBS 2359.

\section{Chemostat cultivations}

We performed two parallel bioreactor cultivations with the K. lactis CBS 2359 strain, using the same defined medium described by Verduyn et al. (1992). This medium was originally designed for $S$. 
cerevisiae, and later adapted by Kiers et al. (1998) to the growth of $K$. lactis, which required an increase in the nicotinic acid concentration from 1 to $5 \mathrm{mg} \mathrm{L}^{-1}$. Both cultivations were initiated with a batch phase, followed by a continuous phase, in which the dilution rate was stepwise increased from 0.1 to $0.2 \mathrm{~h}^{-1}$, $0.3 \mathrm{~h}^{-1}$, and finally to $0.4 \mathrm{~h}^{-1}$. For each dilution rate value, steady state was allowed to be achieved, before measurements were made to verify this condition, and samples were taken. For the different steady states achieved, the main physiological parameters were calculated (Table 1).

According to the data presented in Table 1, it was verified that metabolism was respiratory at dilution rates ranging from 0.1 to $0.4 \mathrm{~h}^{-1}$. The only metabolite detected in the growth medium was glycerol $(0.16 \mathrm{~g}$ glycerol $\mathrm{L}^{-1}$ ) in one of the chemostats, which was running slightly above the desired dilution rate of $0.4 \mathrm{~h}^{-1}\left(0.44 \mathrm{~h}^{-1}\right)$. In this condition, a high residual glucose concentration ( 3.386 g glucose $\mathrm{L}^{-1}$ ) was also observed, indicating that the dilution rate was close to the maximal specific growth rate or that another nutrient other than glucose became growth-limiting at $0.44 \mathrm{~h}^{-1}$, as pointed out by Kiers et al. (1998). While glycerol production in $S$. cerevisiae has been well established and is related with either increased resistance to osmotic stress in environments with low water activity or cofactor balance under oxygen limitation (Nevoigt and Stahl 1997), its excretion in K. lactis has been less studied. In aerobic conditions the glycerol-3phosphate shuttle has been proven to be active for cytoplasmic cofactor balance, also in K. lactis (Saliola et al. 2008). Although it has not been reported, the insufficient activity of the associated KlGUT2 gene at specific growth rates close to the maximum could be speculated.

Overall, the physiological parameters obtained in this study were comparable to those observed by Kiers et al. (1998) in their former study with this same strain. Biomass yield on glucose varied from 0.47 to $0.52 \mathrm{~g}$ DM g glucose ${ }^{-1}$ at all dilution rates evaluated, which was similar to the values reported by other authors for K. lactis CBS 2359 (Kiers et al. 1998; Overkamp et al. 2002). The only exception was observed at a dilution rate of $0.4 \mathrm{~h}^{-1}$, in which biomass yield was 0.48 and $0.46 \mathrm{~g} \mathrm{DM} \mathrm{g}_{\mathrm{glucose}}{ }^{-1}$ in each culture, whereas Kiers et al. 1998 reported a biomass yield of $0.49 \pm 0.01 \mathrm{~g}$ $\mathrm{DM} g$ glucose $^{-1}$ at this dilution rate. This parameter increased slightly when the dilution rate was increased

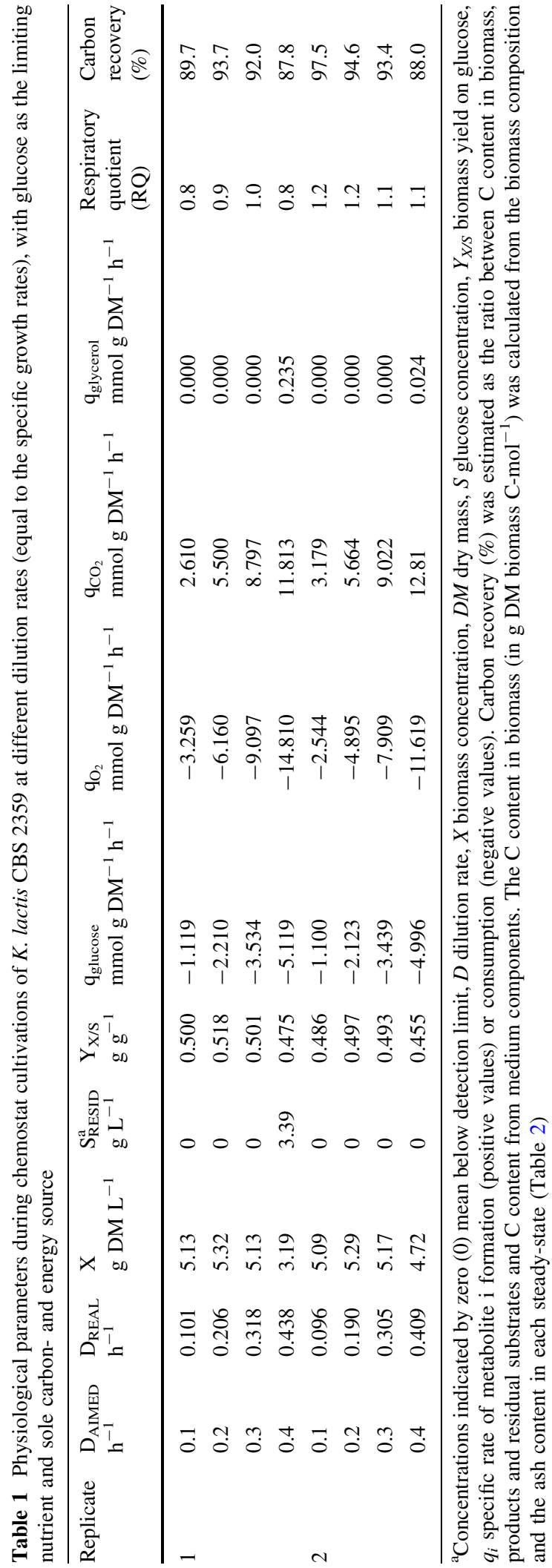


from 0.1 to $0.2 \mathrm{~h}^{-1}$ in view of the higher contribution of maintenance energy requirements at lower growth rates (Pirt 1965). Another indication for fully respiratory metabolism was the value close to 1 of the respiratory quotient (RQ) obtained at all dilution rates (Table 1). Carbon balance also corroborated a purely oxidative metabolism. Both agitation and air flow into the reactor were adjusted manually along the cultivations, since our aim was to keep the cultures under fully aerobic conditions, avoiding oxygen limitation (an automatic control system was not available in our laboratory). Although the agitation and aeration adjustments were not identical in the duplicate experiments, this should not interfere with the microorganism's physiology, due to the fully aerobic condition. This can be proven by the fact that the results obtained in our study corroborate data from a previous study carried out in a completely different laboratory, using a different bioreactor, with different agitation and aeration schemes (Kiers et al. 1998).

It is worth mentioning that the physiological parameters calculated at steady state were also similar to those reported by Inchaurrondo et al. (1998) at a dilution rate of $0.1 \mathrm{~h}^{-1}$, with a different strain (NRRL $\mathrm{y}-1118$ ) and a slightly different defined medium. Specific rates of glucose consumption were $1.11 \pm 0.009 \mathrm{mmol} \mathrm{g} \mathrm{DM}^{-1} \mathrm{~h}^{-1}$ in the present study and $1.26 \mathrm{mmol} \mathrm{g} \mathrm{DM}^{-1} \mathrm{~h}^{-1}$ in the referred work. The biomass yield on glucose was slightly higher in the present study $\left(0.493 \pm 0.007 \mathrm{~g} \mathrm{~g} \mathrm{DM}^{-1}\right)$ when compared to the results reported by the other authors $\left(0.44 \mathrm{~g} \mathrm{~g} \mathrm{DW}^{-1}\right)$. Conversely, specific rates of carbon dioxide production $\left(4.1 \mathrm{mmolg} \mathrm{DM}^{-1} \mathrm{~h}^{-1}\right)$ and oxygen consumption (4.05 mmol g DM ${ }^{-1} \mathrm{~h}^{-1}$ ) were both higher in the reference study when compared to our values $(2.89 \pm 0.28$ and $2.90 \pm 0.36 \mathrm{mmol} \mathrm{g}$ $\mathrm{DM}^{-1} \mathrm{~h}^{-1}$, respectively).

Data consistency of the measured rates of glucose and $\mathrm{O}_{2}$ consumption, cell growth, and $\mathrm{CO}_{2}$ production, together with the elemental composition of $K$. lactis CBS 2359 cell biomass at all dilution rates evaluated, was checked with the software MACROBAL (Hellinga and Romein 1992). According to the software and its statistical hypothesis testing procedure, there was no evidence of gross errors in the experimental dataset. The measured rates and the estimated values calculated with the software MACROBAL are shown in Fig. 1. Experimental data values were within the standard deviation of the estimated data, except for
$\mathrm{CO}_{2}$ and biomass at the highest dilution rate $\left(0.4 \mathrm{~h}^{-1}\right)$. This observation might be explained by the fact that no glycerol production is estimated by the model. In the experiment, as part of the substrate is diverted into glycerol, this leads to diminished rates of both biomass and $\mathrm{CO}_{2}$ production when compared with zero glycerol production.

The elemental composition of biomass during the different steady states achieved during the continuous phase of the cultivations were also determined (Table 2). Chemical analysis of K. lactis CBS 2359 biomass were conducted with cells collected during steady state from carbon-limited chemostat cultures ranging from 0.1 to $0.4 \mathrm{~h}^{-1}$ specific growth rates. The elemental composition of the ash-free yeast biomass displayed some variability among the four dilution rates tested (Table 2). Overall, the elemental composition found in this work for the ash-free biomass of $K$. lactis was to some extent different from that published for S. cerevisiae CEN.PK113-7D (Lange and Heijnen 2001), Dekkera bruxellensis GDB 248 (Leite et al. 2012) and other yeasts as shown in the previously mentioned table. Likewise, the data show a decreasing trend in the carbon to nitrogen ratio, when the dilution rate increases.

We have also calculated the degree of reduction of yeast cell biomass (Heijnen 1981) in K. lactis and in other yeasts reported in the literature. Apparently, the values obtained did not correlate with either the specific growth rate or the yeast species (Table 2). Moreover, oxygen (O) values reported by other authors who did not measure sulphur (S) or phosphorus (P) are overestimated (Cooney et al. 1996; Carnicer et al. 2009), resulting in lower values for the degree of reduction of biomass. These observations highlight the importance of precisely measuring sulphur, phosphorus and ash content in biomass, when a correct estimation of this parameter in cell biomass is to be obtained.

Finally, based on the values estimated with the software MACROBAL and using the biomass compositions given in Table 2, the aerobic growth of $K$ lactis CBS 2359 on glucose at a specific growth rate of $0.1 \mathrm{~h}^{-1}$ could be described by a stoichiometric equation (Eq. 1), and it was compared to the equivalent equation for $S$. cerevisiae CEN.PK113-7D (Eq. 2) grown under similar conditions (Leite et al. 2012). 

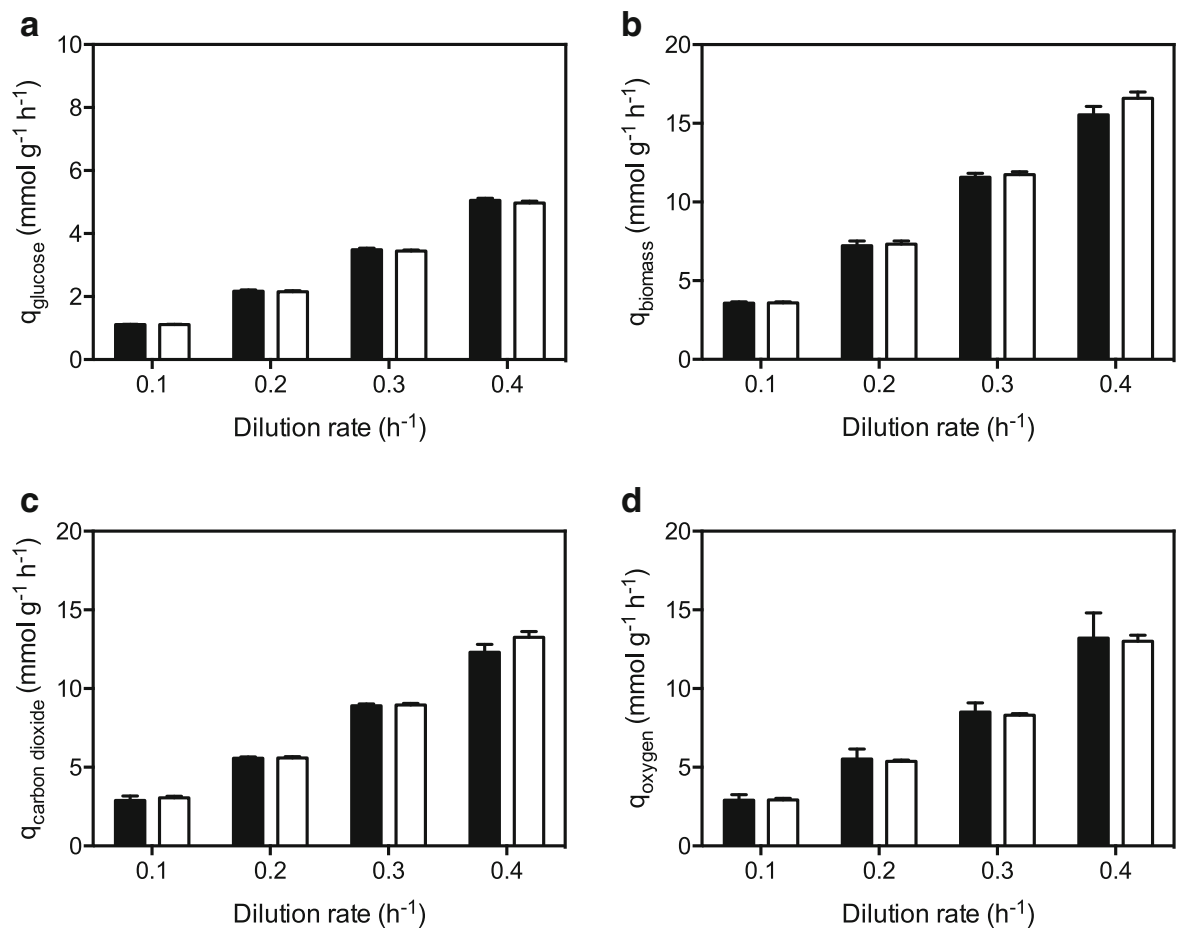

Fig. 1 Experimental and estimated conversion rates in glucoselimited cultures of $K$. lactis. Experimental (black bars) and estimated (white bars) specific rates (in $\mathrm{mmol} \mathrm{g}^{-1} \mathrm{~h}^{-1}$ ) of glucose consumption (a), biomass formation (b), $\mathrm{CO}_{2}$ formation (c) and $\mathrm{O}_{2}$ consumption (d). Whereas the error bars for the measured conversion rates (back bars) represent mean

$$
\begin{aligned}
& \mathrm{C}_{6} \mathrm{H}_{12} \mathrm{O}_{6}+0.42 \mathrm{NH}_{3}+2.64 \mathrm{O}_{2} \\
& \quad \rightarrow 3.24 \mathrm{CH}_{1.98} \mathrm{O}_{0.72} \mathrm{~N}_{0.13}+2.76 \mathrm{CO}_{2}+3.46 \mathrm{H}_{2} \mathrm{O} \\
& \text { K. lactis }
\end{aligned}
$$

$$
\begin{aligned}
& \mathrm{C}_{6} \mathrm{H}_{12} \mathrm{O}_{6}+0.53 \mathrm{NH}_{3}+2.31 \mathrm{O}_{2} \\
& \quad \rightarrow 3.59 \mathrm{CH}_{1.75} \mathrm{O}_{0.60} \mathrm{~N}_{0.15}+2.41 \mathrm{CO}_{2}+3.66 \mathrm{H}_{2} \mathrm{O} \\
& \text { S. cerevisiae }
\end{aligned}
$$

\section{Discussion}

The batch cultivation performed in duplicate in the present work, using the same strain and cultivation medium previously employed by Kiers et al. (1998) yielded very similar growth parameters, mainly the specific growth rate and extracellular metabolite formation, when compared to the latter work. This

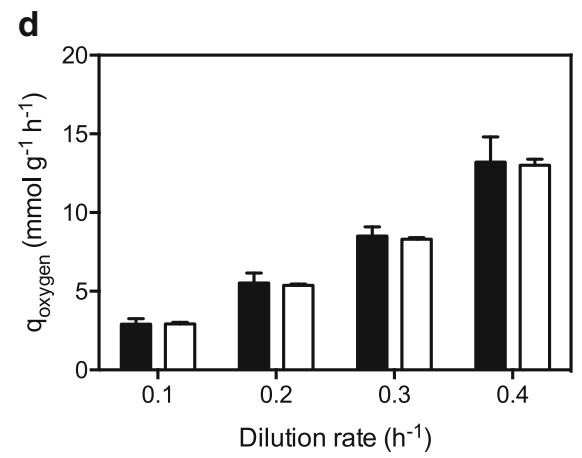

deviations, the error bars for estimated rates (white bars) represent standard deviations. Steady-state conversion rates were analyzed for data consistency using the software MACROBAL 2.02, obtained from the Delft University of Technology (Hellinga and Romein 1992)

observation served as a validation for our experimental setup, which we considered necessary to perform, before starting the chemostat experiments. Although the same strain and cultivation medium were employed in both works, the chemicals were purchased from different suppliers, the bioreactors, the experimenters and the inoculation strategy were different, which could have led to different results. Once this validation was made, the chemostat experiments were designed and performed.

Data on the growth of $K$. lactis using a combination of chemostat cultivation and completely defined media are scarce in the literature. This type of approach is essential for understanding the nutritional requirements of $K$. lactis at different growth rates and also to elucidate the dependency of key biological processes on the growth rate. Kiers et al. (1998) demonstrated that the defined medium previously developed by Verduyn et al. (1992) for S. cerevisiae was suitable for fully respiratory growth of $K$. lactis 


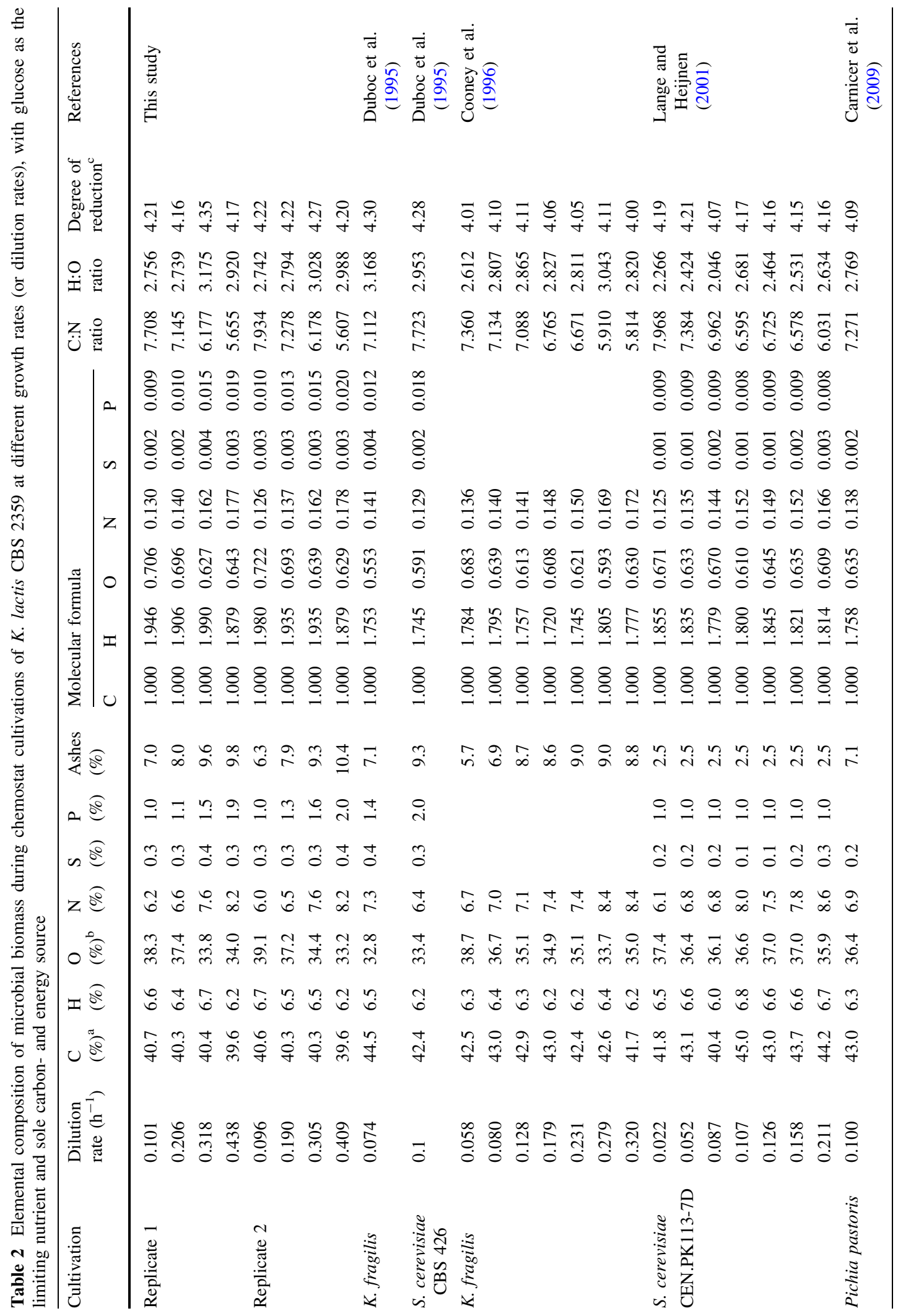


CBS 2359, as long as the nicotinic acid concentration was increased from 1 to $5 \mathrm{mg} \mathrm{L}^{-1}$. This increase was necessary to keep the fully respiratory metabolism of $K$. lactis operating at growth rates as high as $0.4 \mathrm{~h}^{-1}$. This special requirement for nicotinic acid is corroborated by the work of Inchaurrondo et al. (1998), who cultivated $K$. lactis NRRL y-1118 (a strain that has a stronger glucose repression phenotype than the NRRL y-1140 = CBS 2359 strain) in chemostat mode under fully aerobic conditions, on a synthetic medium with $12 \mathrm{mg} \mathrm{L}^{-1}$ nicotinic acid, observing fully respiratory metabolism up to $0.5 \mathrm{~h}^{-1}$. The cause for this phenomenon was assigned to the absence of a de novo biosynthetic pathway for NAD in K. lactis ( $\mathrm{Li}$ and Bao 2007).

The fact that the nutritional requirement for nicotinic acid of chemostat growing K. lactis CBS 2359 cells is different at varying dilution rates, indicates that the biomass composition of $K$. lactis CBS 2359 is not the same at different dilution rates (Kiers et al. 1998). Although this was already pointed out in the latter work, the biomass composition was not studied. Inchaurrondo et al. (1998) measured the macromolecular composition of biomass during chemostat cultivations of $K$. lactis NRRL y-1118, in terms of total carbohydrate and protein content. They observed that protein content did not change (49\%; relative to dried biomass) when increasing dilution rate from 0.1 to $0.25 \mathrm{~h}^{-1}$ in aerobic glucose-limited cultures, whereas total carbohydrate in biomass decreased from 30 to $29 \%$ after this shift.

The elemental composition of biomass is essential for performing carbon balances, and for metabolic flux analysis or metabolic modelling studies, for which a "molecular formula" for the biomass is required (Lange and Heijnen 2001). The elemental composition of a given microorganism is dependent on the strain and the growth conditions. While $\mathrm{N}$ content is highly influenced by growth conditions, $\mathrm{S}$ and $\mathrm{P}$ might lead to inaccurate $\mathrm{O}$ determinations if they are not determined experimentally. This, in turn, might result in variations regarding the mass of $1 \mathrm{C}$-mol biomass for a particular strain and a given condition (Duboc et al. 1995). The work performed by Duboc et al. (1995), one of the first studies to raise awareness over accurate determination of biomass elemental composition, described Kluyveromyces fragilis (K. marxianus) sampled from a continuous culture in a semi-defined medium, as indicated in Table 2. According to these authors, 
while $\mathrm{C}$ and $\mathrm{H}$ contents varied only slightly from one microorganism to another, ash, $\mathrm{N}, \mathrm{P}$, and $\mathrm{S}$ mass fractions varied considerably. This is reflected by the variability present in the macromolecule content, such as protein, carbohydrates and RNA.

Soon after, it was shown that mass fractions of N, P, and ash in $K$. fragilis increased almost linearly, eventually reaching a plateau, with the dilution rate in carbon-limited chemostats (Cooney et al. 1996). Under glucose-limited cultures, Cooney et al. (1996) showed that the $\mathrm{N}$ content increased with dilution rate from 6.7 to $8.4 \%$, probably as a result of increased protein synthesis. These results are in close agreement to our data for $K$. lactis (Table 2). A similar behaviour was observed for the ash content, while the $\mathrm{C}$ and $\mathrm{H}$ contents remained virtually unchanged with increasing dilution rates. Overall, these authors concluded that the use of an average elemental composition for different growth conditions may lead to incorrect metabolic modeling calculations.

When comparing our results to those obtained with other yeast genera, elemental biomass composition of S. cerevisiae under glucose-limited chemostat cultures, reported by Lange and Heijnen (2001), resulted in similar trends as observed in the present investigation, using $K$. lactis (Table 2). $\mathrm{N}$ content increased from 6.1 to $8.6 \%$ when dilution rate was increased from 0.02 to $0.21 \mathrm{~h}^{-1}$. In accordance to our results, $\mathrm{H}$ content did not exhibit a clear trend and values remained around $6.5 \%$. The only difference observed was related to the $\mathrm{C}$ content, that increased from 42 to $44 \%$ when the dilution rate was increased from 0.02 to $0.21 \mathrm{~h}^{-1}$, while in our study, it remained steady at $40 \%$ as dilution rate increased from 0.1 to $0.4 \mathrm{~h}^{-1}$. In addition to that, the average degree of reduction of biomass in $S$. cerevisiae $(4.16 \pm 0.05)$ was very close to K. lactis (4.22 \pm 0.06$)$. We must emphasize that, in their work, Lange and Heijnen (2001) kept the culture always below the critical dilution rate for $S$. cerevisiae, i.e. at an entirely (fully) oxidative metabolism.

Finally, Carnicer et al. (2009) performed an extensive analysis of the elemental biomass composition of Pichia pastoris under varying conditions of oxygen concentration in the inlet gas of carbon-limited chemostat cultures. They highlighted that availability of such data is scarce or even inexistent for a specific strain or species and growth condition, particularly in non-model organisms. When we compare their data, under normoxic conditions of oxygen supply $\left(21 \% \mathrm{O}_{2}\right.$ in the inlet gas at $1.5 \mathrm{vvm}$ ) at a dilution rate of $0.1 \mathrm{~h}^{-1}$, with our results, $P$. pastoris elemental biomass composition presents a higher mass fraction of the elemental compounds $\mathrm{C}(43.0 \%)$ and $\mathrm{N}(6.9 \%)$ than the values observed in $K$. lactis in the present study ( $40.7 \%$ for $\mathrm{C}$ and $6.1 \%$ for $\mathrm{N}$ ). Oleaginous yeasts, such as Rhodosporidium toruloides, display an even higher fraction of $\mathrm{C}(47.0 \%)$ in biomass under carbon limitation at similar dilution rates, and consequently a high degree of reduction of biomass (4.29), presumably due to lipid accumulation (Minkevich et al. 2010). Recently, it has been reported that there was almost no difference in the elemental composition of $R$. toruloides biomass at varying dilution rates (Shen et al. 2017).

In the experiments performed here, four steadystates were achieved, each one after 5 volume changes. This corresponds to roughly 28 generations from the beginning of the continuous phase until the final steady state. While it is not possible to completely exclude that evolution might have taken place at some stage during these cultivations, reported data indicate that macroscopic fluxes (such as biomass concentration, specific consumption rates of substrate and oxygen and specific carbon dioxide production) do not change during up to 90 generations in prolonged glucose-limited chemostat cultivations using $S$. cerevisiae (Mashego et al. 2005). These observations in macroscopic parameters were also corroborated by Jansen et al. (2005), when $S$. cerevisiae was kept for 200 generations in long-term glucose-limited chemostat cultures. Moreover, measurements of intracellular metabolites and enzyme activities (that might reflect in cellular protein concentration) in such experiments demonstrated that major changes occur only after 40-50 generations have passed (Jansen et al. 2005; Mashego et al. 2005). Therefore, from a macroscopic point of view, we may exclude any changes in the measured and calculated parameters in our 28-generations study, due to an eventual evolutionary process.

Since our aim was to determine the elemental composition of biomass and the major adaptation that occurs to yeast cells growing in prolonged chemostats is an improved affinity for the limiting substrate, which already before adaptation presents very low concentrations, we expect that an eventual adaptation will not affect the elemental biomass composition of the cells. Furthermore, transcriptome analysis (Ferea et al. 1999; Jansen et al. 2005) and changes in DNA 
copy number (Dunham et al. 2002) as well as point mutations (Gresham et al. 2008) have been used to study microbial evolution in chemostat cultures. But for that, yeast strains had to undergo more than 100-200 generations in glucose-limited chemostat cultures.

We believe that the data presented here will aid researchers in deepening their knowledge on the yeast $K$. lactis and on yeast in general, besides providing material for setting up metabolic models for this microorganism, which can have several purposes, e.g. in performing metabolic network analysis (Gombert and Nielsen 2000).

Acknowledgements This study was supported by the Portuguese Foundation for Science and Technology (FCT) under the scope of the strategic funding of UID/BIO/04469/2013 unit and COMPETE 2020 (POCI-01-0145-FEDER-006684) and BioTecNorte operation (NORTE-01-0145-FEDER-000004) funded by the European Regional Development Fund under the scope of Norte2020 - Programa Operacional Regional do Norte. T.O.B. would like to express his gratitude for funds provided by Coordenação de Aperfeiçoamento de Pessoal de Nível Superior (CAPES, Brasília, Brazil).

Conflict of interest The authors declare that they have no conflict of interest.

\section{References}

Basso TO, Gomes FS, Lopes ML, et al (2014) Homo- and heterofermentative lactobacilli differently affect sugarcane-based fuel ethanol fermentation. Antonie Van Leeuwenhoek 105:169-177. doi:10.1007/s10482-0130063-6

Becerra M, Tarrío N, González-Siso MI, Cerdán ME (2004) Genome-wide analysis of Kluyveromyces lactis in wildtype and rag 2 mutant strains. Genome 47:970-978. doi:10. 1139/g04-039

Blondeau K, Boutur O, Boze H, Moulin G, Galzy P (1993) Influence of culture conditions on the production of heterologous interleukin 1 ? by Kluyveromyces lactis. Biotechnol Tech 7:609-614. doi:10.1007/BF00156338

Bonekamp FJ, Oosterom J (1994) On the safety of Kluyveromyces lactis-A review. Appl Microbiol Biotechnol 41:1-3

Breunig K, Bolotin-Fukuhara M, Bianchi M, Bourgarel D, Falcone C, Ferrero I, Frontali L, Goffrini P, Krijger J, Mazzoni C, Milkowski C, Steensma H, Wésolowski-Louvel M, Zeeman A (2000) Regulation of primary carbon metabolism in Kluyveromyces lactis. Enzyme Microb Technol 26:771-780. doi:10.1016/S0141-0229(00)00170-8

Carnicer M, Baumann K, Töplitz I, Sánchez-Ferrando F, Mattanovich D, Ferrer P, Albiol J (2009) Macromolecular and elemental composition analysis and extracellular metabolite balances of Pichia pastoris growing at different oxygen levels. Microb Cell Fact 8:65. doi:10.1186/14752859-8-65

Clark-Walker D (2006) Eighteenth biology of Kluyveromyces meeting, Bratislava, Slovakia, 6 August 2005. FEMS Yeast Res 6:141-141. doi:10.1111/j.1567-1364.2005.00044.x

Cooney MJ, Marison IW, van Gulik WM, von Stockar U (1996) Calorimetric and stoichiometric analysis of growth of Kluyveromices fragilis in continuous culture: nitrogen limitation imposed upon carbon-limited growth. Appl Microbiol Biotechnol 44:643-653. doi:10.1007/ BF00172498

Dias O, Pereira R, Gombert AK, Ferreira EC, Rocha I (2014) iOD907, the first genome-scale metabolic model for the milk yeast Kluyveromyces lactis. Biotechnol J 9:776-790. doi:10.1002/biot.201300242

Dickson RC, Barr K (1983) Characterization of lactose transport in Kluyveromyces lactis. J Bacteriol 154:1245-1251

Dickson RC, Markin JS (1980) Physiological studies of betagalactosidase induction in Kluyveromyces lactis. J Bacteriol 142:777-785

Dickson RC, Dickson LR, Markin JS (1979) Purification and properties of an inducible beta-galactosidase isolated from the yeast Kluyveromyces lactis. J Bacteriol 137:51-61

Duboc P, Schill N, Menoud L, van Gulik W, von Stockar U (1995) Measurements of sulfur, phosphorus and other ions in microbial biomass: influence on correct determination of elemental composition and degree of reduction. J Biotechnol 43:145-158

Dujon B, Sherman DJ, Fischer G, Durrens P, Casaregola S, Lafontaine I, de Montigny J, Marck C, Neuveglise C, Talla $E$ et al (2004) Genome evolution in yeasts. Nature 430:435-444

Dunham MJ, Badrane H, Ferea T, Adams J, Brown PO, Rosenzweig F, Botstein D (2002) Characteristic genome rearrangements in experimental evolution of Saccharomyces cerevisiae. Proc Natl Acad Sci USA 99:16144-16149. doi:10.1073/pnas.242624799

Ferea TL, Botstein D, Brown PO, Rosenzweig RF (1999) Systematic changes in gene expression patterns following adaptive evolution in yeast. Proc Natl Acad Sci 96:9721-9726. doi:10.1073/pnas.96.17.9721

Fonseca GG, Heinzle E, Wittmann C, Gombert AK (2008) The yeast Kluyveromyces marxianus and its biotechnological potential. Appl Microbiol Biotechnol 79:339-354. doi:10. 1007/s00253-008-1458-6

Fukuhara H, Boekhout T (2007) Thematic issue on Kluyveromyces lactis. FEMS Yeast Res 7:641-641. doi:10.1111/j. 1567-1364.2007.00277.x

Gombert AK, Nielsen J (2000) Mathematical modelling of metabolism. Curr Opin Biotechnol 11:180-186

González-Siso M, Freire-Picos M, Ramil E, González-Domínguez M, Rodríguez Torres A, Cerdán M (2000) Respirofermentative metabolism in Kluyveromyces lactis. Enzyme Microb Technol 26:699-705. doi:10.1016/S01410229(00)00161-7

González-Siso MI, García-Leiro A, Tarrío N, Cerdán ME (2009) Sugar metabolism, redox balance and oxidative stress response in the respiratory yeast Kluyveromyces lactis. Microb Cell Fact 8:46. doi:10.1186/1475-2859-8-46 
Gresham D, Desai MM, Tucker CM, Jenq HT, Pai DA, Ward A, DeSevo CG, Botstein D, Dunham MJ (2008) The repertoire and dynamics of evolutionary adaptations to controlled nutrient-limited environments in yeast. PLoS Genet. doi:10.1371/journal.pgen.1000303

Heijnen JJ (1981) Application of the macroscopic electric charge balance in fermentation modeling. Biotechnol Bioeng 23:1133-1144. doi:10.1002/bit.260230520

Hellinga C, Romein B (1992) MACROBAL: A Program for Robust Data Reconciliation and Gross Error Detection. In: Model Control Biotech Process. pp 459-460

Hensing MCM, Bangma KA, Raamsdonk L, de Hulster E, van Dijken JP, Pronk JT (1995) Effects of cultivation conditions on the production of heterologous alpha-galactosidase production by Kluyveromyces lactis

Hoekstra R, Groeneveld P, van Verseveld HW, Stouthamer AH, Planta RJ (1994) Transcription regulation of ribosomal protein genes at different growth rates in continuous cultures of Kluyveromyces yeasts. Yeast 10:637-651. doi:10. 1002/yea.320100508

Inchaurrondo VAA, Flores MVV, Voget CEE (1998) Growth and beta-galactosidase synthesis in aerobic chemostat cultures of Kluyveromyces lactis. J Ind Microbiol Biotechnol 20:291-298. doi:10.1038/sj.jim.2900526

Jansen MLA, Diderich JA, Mashego M, Hassane A, de Winde JH, Daran-Lapujade P, Pronk JT (2005) Prolonged selection in aerobic, glucose-limited chemostat cultures of Saccharomyces cerevisiae causes a partial loss of glycolytic capacity. Microbiology 151:1657-1669. doi:10. 1099/mic.0.27577-0

Kiers J, Zeeman A-MM, Luttik M, Thiele C, Castrillo JI, Steensma HY, Van Dijken JP, Pronk JT (1998) Regulation of alcoholic fermentation in batch and chemostat cultures of Kluyveromyces lactis CBS 2359. Yeast 14:459-469. doi:10.1002/(SICI)1097-0061(19980330)14:5<459:AIDYEA248>3.0.CO;2-O

Kurtzman CP (2003) Phylogenetic circumscription of Saccharomyces, Kluyveromyces and other members of the Saccharomycetaceae, and the proposal of the new genera Lachancea, Nakaseomyces, Naumovia, Vanderwaltozyma and Zygotorulaspora. FEMS Yeast Res 4:233-245. doi:10. 1016/S1567-1356(03)00175-2

Lane MM, Morrissey JP (2010) Kluyveromyces marxianus: a yeast emerging from its sister's shadow. Fungal Biol Rev 24:17-26. doi:10.1016/j.fbr.2010.01.001

Lange HC, Heijnen JJ (2001) Statistical reconciliation of the elemental and molecular biomass composition of Saccharomyces cerevisiae. Biotechnol Bioeng 75:334-344. doi:10.1002/bit.10054

Leite FCB, Basso TO, Pita WB, Gombert AK, Simões DA, de Morais Júnior MA (2012) Quantitative aerobic physiology of the yeast Dekkera bruxellensis, a major contaminant in bioethanol production plants. FEMS Yeast Res. doi:10. 1111/j.1567-1364.2012.12007.x

Li Y-F, Bao W-G (2007) Why do some yeast species require niacin for growth? Different modes of NAD synthesis. FEMS Yeast Res 7:657-664. doi:10.1111/j.1567-1364. 2007.00231.X

Mashego MR, van Gulik WM, Vinke JL, Heijnen JJ (2003) Critical evaluation of sampling techniques for residual glucose determination in carbon-limited chemostat culture of Saccharomyces cerevisiae. Biotechnol Bioeng 83:395-399. doi:10.1002/bit.10683

Mashego MR, Jansen MLA, Vinke JL, Van Gulik WM, Heijnen JJ (2005) Changes in the metabolome of Saccharomyces cerevisiae associated with evolution in aerobic glucoselimited chemostats. FEMS Yeast Res 5:419-430. doi:10. 1016/j.femsyr.2004.11.008

Minkevich IG, Dedyukhina EG, Chistyakova TI (2010) The effect of lipid content on the elemental composition and energy capacity of yeast biomass. Appl Microbiol Biotechnol 88:799-806. doi:10.1007/s00253-010-2766-1

Nevoigt E, Stahl U (1997) Osmoregulation and glycerol metabolism in the yeast Saccharomyces cerevisiae. FEMS Microbiol Rev 21:231-241

Oliveira GA, Tahara EB, Gombert AK, Barros MH, Kowaltowski AJ (2008) Increased aerobic metabolism is essential for the beneficial effects of caloric restriction on yeast life span. J Bioenerg Biomembr 40:381-388. doi:10.1007/ s10863-008-9159-5

Olsson L, Nielsen J (1997) On-line and in situ monitoring of biomass in submerged cultivations. Trends Biotechnol 15:517-522. doi:10.1016/S0167-7799(97)01136-0

Overkamp KM, Bakker BM, Steensma HY, van Dijken JP, Pronk JT (2002) Two mechanisms for oxidation of cytosolic NADPH by Kluyveromyces lactis mitochondria. Yeast 19:813-824. doi:10.1002/yea.878

Pirt SJ (1965) The maintenance energy of bacteria in growing cultures. Proc R Soc Lond B Biol Sci 163:224-231

Rocha SN, Abrahão-Neto J, Cerdán ME, Gombert AK, González-Siso MI (2011) Heterologous expression of a thermophilic esterase in Kluyveromyces yeasts. Appl Microbiol Biotechnol 89:375-385. doi:10.1007/s00253010-2869-8

Roux AE, Chartrand P, Ferbeyre G, Rokeach LA (2010) Fission yeast and other yeasts as emergent models to unravel cellular aging in eukaryotes. J Gerontol A Biol Sci Med Sci 65:1-8. doi:10.1093/gerona/glp152

Saliola M, Sponziello M, D'Amici S, Lodi T, Falcone C (2008) Characterization of KlGUT2, a gene of the glycerol-3phosphate shuttle, in Kluyveromyces lactis. FEMS Yeast Res 8:697-705. doi:10.1111/j.1567-1364.2008.00386.x

Schaffrath R, Breunig KD (2000) Genetics and Molecular Physiology of the Yeast Kluyveromyces lactis. Fungal Genet Biol 30:173-190. doi:10.1006/fgbi.2000.1221

Shen H, Zhang X, Gong Z, Wang Y, Yu X, Yang X, Zhao ZK (2017) Compositional profiles of Rhodosporidium toruloides cells under nutrient limitation. Appl Microbiol Biotechnol 101:3801-3809. doi:10.1007/s00253-0178157-0

Sluiter A, Hames B, Ruiz R, Scarlata C, Sluiter J, Templeton D (2005) Determination of ash in biomass: laboratory analytical procedure (LAP). Natl Renew Energy Lab

Souciet J-LJ-L, Dujon B, Gaillardin C, Johnston M, Baret PV, Cliften P, Sherman DJ, Weissenbach J, Westhof E, Wincker P, Jubin C, Poulain J, Barbe V, Segurens B, Artiguenave F, Anthouard V, Vacherie B, Val M-EM-EME, Fulton RS, Minx P, Wilson R, Durrens P, Jean G, Marck C, Martin T, Nikolski M, Rolland T, Seret M-LM-LM-L, Casaregola S, Despons L, Fairhead C, Fischer G, Lafontaine I, Leh V, Lemaire M, de Montigny J, Neuveglise C, Thierry A, Blanc-Lenfle I, Bleykasten C, Diffels J, Fritsch 
E, Frangeul L, Goeffon A, Jauniaux N, Kachouri-Lafond R, Payen C, Potier S, Pribylova L, Ozanne C, Richard G-FG-F, Sacerdot C, Straub M-LM-L, Talla E, Ségurens B, Artiguenave F, Anthouard V, Vacherie B, Val M-EM-EME, Fulton RS, Minx P, Wilson R, Durrens P, Jean G, Marck C, Martin T, Nikolski M, Rolland T, Seret M-LM-LM-L, Casarégola S, Despons L, Fairhead C, Fischer G, Lafontaine I, Leh V, Lemaire M, de Montigny J, Neuvéglise C, Thierry A, Blanc-Lenfle I, Bleykasten C, Diffels J, Fritsch E, Frangeul L, Goëffon A, Jauniaux N, Kachouri-Lafond R, Payen C, Potier S, Pribylova L, Ozanne C, Richard G-FG-F, Sacerdot C, Straub M-LM-L, Talla E, Segurens B, Artiguenave F, Anthouard V, Vacherie B, Val M-EM-EME, Fulton RS, Minx P, Wilson R, Durrens P, Jean G, Marck C, Martin T, Nikolski M, Rolland T, Seret M-LM-LM-L, Casaregola S, Despons L, Fairhead C, Fischer G, Lafontaine I, Leh V, Lemaire M, de Montigny J, Neuveglise C, Thierry A, Blanc-Lenfle I, Bleykasten C, Diffels J, Fritsch E, Frangeul L, Goeffon A, Jauniaux N, Kachouri-Lafond R, Payen C, Potier S, Pribylova L, Ozanne C, Richard G-FG-F, Sacerdot C, Straub M-LM-L, Talla E (2009) Comparative genomics of protoploid Saccharomycetaceae. Genome Res 19:1696-1709. doi:10.1101/gr. 091546.109

Spohner SC, Schaum V, Quitmann H, Czermak P (2016) Kluyveromyces lactis: an emerging tool in biotechnology.
J Biotechnol 222:104-116. doi:10.1016/j.jbiotec.2016.02. 023

Suleau A, Gourdon P, Reitz-Ausseur J, Casaregola S (2006) Transcriptomic analysis of extensive changes in metabolic regulation in Kluyveromyces lactis strains. Eukaryot Cell 5:1360-1370. doi:10.1128/EC.00087-06

van Ooyen AJJJ, Dekker P, Huang M, Olsthoorn MMAA, Jacobs DI, Colussi PA, Taron CH (2006) Heterologous protein production in the yeast Kluyveromyces lactis. FEMS Yeast Res 6:381-392. doi:10.1111/j.1567-1364. 2006.00049.x

Verduyn C, Postma E, Scheffers WA, Van Dijken JP (1992) Effect of benzoic acid on metabolic fluxes in yeasts: a continuous-culture study on the regulation of respiration and alcoholic fermentation. Yeast 8:501-517. doi:10.1002/ yea.320080703

Walsh DJ, Gibbs MD, Bergquist PL (1998) Expression and secretion of a xylanase from the extreme thermophile, thermotoga strain FjSS3B.1, in Kluyveromyces lactis. Extremophiles 2:9-14

Wésolowski-Louvel M, Breunig KD, Fukuhara H (1996) Kluyveromyces lactis. Nonconventional Yeasts in Biotechnology. Springer, Berlin Heidelberg, pp 139-201

(2016) Go forth and replicate! Nature 536:373-373. doi: 10. $1038 / 536373 a$ 